\title{
A Comparison of the Boolean and Continuous Dynamics of Three-Gene Regulatory Networks
}

\author{
Timothy D. Comar ${ }^{1,}{ }^{*}$, Marihan Hegazy ${ }^{1}$, Miranda Henderson ${ }^{1}$, \\ Daniel Hrozencik ${ }^{2}$
}

\begin{abstract}
We investigate the dynamics of three-gene regulatory networks with one feedback circuit using the Boolean and continuous models put forth by Gehrmann and Drossel [4]. We establish the existence of Hopf bifurcations in the continuous models and use these bifurcations to compare the models more closely. With this analysis we are able to establish the regions in the parameter space where the dynamical behavior of the models agree and where they disagree.
\end{abstract}

Keywords: gene regulatory network, Boolean dynamics, bifurcations

\section{Introduction}

Genes are responsible for the production of proteins in cells. The process of gene expression begins with the DNA being transcribed from DNA to messenger RNA (mRNA) within a cell's nucleus. After transcription, the mRNA leaves the cell nucleus and is translated into proteins by ribosomes in the cytoplasm. The protein produced through these processes can then influence the expression of other genes by activating or inhibiting the next gene in a sequence, or even itself. Activation will allow the genes to be expressed, while inhibition stops the gene from being expressed. Networks of genes can be represented by wiring diagrams that describe how the genes influence one another. These wiring diagrams represent gene regulatory networks, which describe how the expression of the genes influence by one another. An example of a gene regulatory network and the corresponding diagram of the macromolecules involved can be seen in Figure 1.

Occasionally in these regulatory networks, feedback circuits, which are containing loops between one or more genes, may be present. These feedback circuits can be assigned a sign depending on the number of activators and inhibitors involved in the circuit. Activators are assigned +1 and inhibitors -1 . By multiplying the sign(s) of the activator(s) and inhibitor(s), the sign of the circuit can be determined as positive or negative. In 1981, $\mathrm{R}$. Thomas [14] proposed how dynamical behavior was determined by the sign of the feedback circuits. In particular, he postulated the following:

1. A necessary condition for multistability (i.e., the existence of several stable fixed points in the dynamics) is the existence of a positive circuit in the regulatory network.

2. A necessary condition for the existence of an attractive cycle in the dynamics is the existence of a negative circuit.

\footnotetext{
${ }^{2}$ Department of Mathematics, Benedictine University, Lisle, IL

${ }^{2}$ Chicago State University, Chicago, IL

${ }^{*}$ Correspondence: tcomar@ben.edu
} 


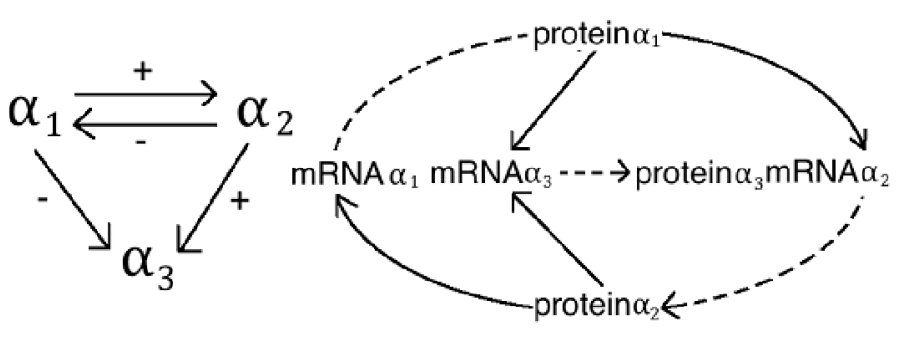

Figure 1: On the left, a diagram of the macromolecules involved in gene expression is illustrated. Solid lines represent the transcription of mRNA, while dashed lines depict the translation of the corresponding protein. On the right is a picture of an interaction graph of a three-gene regulatory network that describes how genes influence one another.

Thomas' conjectures for gene regulatory networks were proven for networks with only one circuit [13]. Additional results for gene regulatory networks with a single circuit were developed using symbolic steady states $[12,11]$.

Due to the interaction of genes, understanding the behavior of small gene networks is essential before trying to work with larger networks that represent complex biological systems. In this paper, we investigate the dynamics of three-gene regulatory networks with one feedback circuit which is not a self-circuit. We will look at both continuous and synchronous Boolean models of the network and establish conditions on where in parameter space these different models give rise to consistent dynamical behavior. We also investigate whether the sign of the circuit influences the dynamics of these networks.

Several authors have investigated similar problems previously. Gehrmann and Drossel [4] looked at two-gene models with two connections and with three connections, one of which is a self-circuit. In their paper, they use generalized models and the method of resultants introduced by Gross and Feudel $[5,6]$ to establish general conditions for the onset of Hopf bifurcations. Their description uses only the signs of the circuits as well as the Hill exponent and the time-scale ratio of the mRNA and protein dynamics, and is the first to use all of these parameters. They conclude that the occurrence of a cycle in the Boolean dynamics is neither necessary nor sufficient for an oscillation in the continuous dynamics. Their work ties together and expands upon the work of others $[2,7,10,17]$ in the realm of two-gene networks. Our work differs in that we consider larger networks (three-gene networks) with more connections (four), and neither self-circuits nor Hamiltonian circuits. Interestingly we obtain similar results to Gehrmann and Drossel [4].

Previous work on networks larger than two genes has been conducted by Norrell [9] and Mochizuki [8]. Norrell investigated four-gene networks with two circuits, one of which is Hamiltonian and one additional self-circuit. Their model only keeps track of translation, not transcription. They do not perform a bifurcation analysis, but they do recognize the importance of the Hill exponents in the dynamics of their continuous models. Mochizuki [8] has looked at random networks with larger numbers of nodes. He reaches several important conclusions. First, there is a direct relation between the number of steady states and the number of self-circuits in the network. Secondly, the dynamics of the Boolean models contain periodic orbits that are not seen in the continuous models, making the Boolean models suspect for predicting dynamical behavior of larger networks.

In this paper, we are particularly interested in three-gene networks with one feedback circuit and exactly one gene governed by a logical gate. The dynamics of the Boolean model is presented in Section 2. Numerical results for the the continuous model are described in Section 3. In Section 4, we perform stability analysis of the continuous model, which involves the determination of Hopf bifurcations in the parameter space. 


\section{Synchronous Updating Boolean Model}

A wiring diagram, where nodes are genes and edges define the interactions among the genes, characterizes a gene regulatory network. In the Boolean model, genes are recognized as either ON (1) or OFF (0). This model reduces the quantitative complexity of the gene interactions to the qualitative, logical structure of the network.

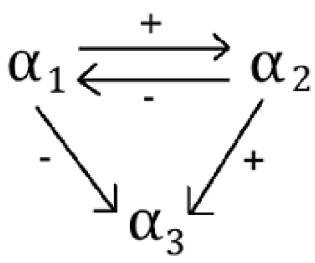

Figure 2: Example interaction graph for a three-gene regulatory network.

Definition 2.1. Let $\alpha_{1}, \alpha_{2}, \ldots, \alpha_{n}$ be the genes in a network. A state $x$ is an ordered $n$-tuple $\left(x_{1}, x_{2}, \ldots, x_{n}\right)$ in which $x_{i}$ is either 0 or 1 depending on whether gene $\alpha_{i}$ is OFF or ON, respectively. We denote the state space by $S=0,1^{n}$.

Algebraically, we consider $S$ as an $n$-dimensional vector space over the field of order 2 .

Definition 2.2. A gene regulatory network $(G, f)$ consists of a set of genes $\alpha_{1}, \alpha_{2}, \ldots, \alpha_{n}$ which can be represented using an interaction graph and a state transition function $f=$ $\left(f_{1}, f_{2}, \ldots, f_{n}\right): S \quad S$.

By assigning each gene a state, note that there are $2^{n}$ possible states, where $n$ represents the number of genes in the interaction graph. A sample interaction graph is depicted in Figure 2 . The $2^{n}$ states can be represented by a state transition graph.

Definition 2.3. A state transition graph of a gene regulatory network $(G, f)$ is a directed graph with vertices for each of the $2^{n}$ states in the state space $S$. There is an edge from a state $x_{i} \quad S$ to $x_{j} \quad S$ if and only if $f\left(x_{i}\right)=x_{j}$.

The occurrence of fixed points and state cycles describes the qualitative behavior of these networks. By constructing state transition graphs for each of the small gene regulatory networks, preliminary results are produced based on the characteristics found in the interaction graphs for each network.

In the event of two genes acting upon a single gene, we consider three logical gates: AND, XOR, and OR. If the logical gate is AND, then both genes will influence the gene they act upon together. When the logical gate is XOR, then one gene or the other, not both, will influence the gene being acted upon. The logical gate OR follows from any of the previous scenarios occurring, that is one, the other, or both can possibly influence the gene. Assuming genes $\alpha_{i}$ and $\alpha_{j}$ activate gene $\alpha_{1}$, the mathematical representations of these logical gates are

$$
\begin{aligned}
\text { AND: } f_{1}\left(x_{1}, x_{2}, \ldots, x_{n}\right) & =x_{i} x_{j}, \\
\text { XOR: } f_{1}\left(x_{1}, x_{2}, \ldots, x_{n}\right) & =x_{i}+x_{j}, \\
\text { OR: } f_{1}\left(x_{1}, x_{2}, \ldots, x_{n}\right) & =x_{i}+x_{j}+x_{i} x_{j} .
\end{aligned}
$$

If $\alpha_{i}$ is an inhibitor, then $x_{i}$ is replaced by $1+x_{i}$. To see this, if $x_{i}=1$, then $1+x_{i}=$ $1+1=0$, which has the effect of inhibition on the gene it influences. Similarly, if $x_{i}=0$, then $1+x_{i}=1+0=1$, which allows the influenced gene to remain active. Based on the 
genes influence on one another in the interaction graph, a corresponding function can be written for each gene, including the gene with the logical gate.

In this paper, we compare the qualitative behavior of Boolean models and continuous models for particular small gene regulatory networks. The following two theorems summarize the relationship between the sign of the feedback circuit and the Boolean dynamics for the three-gene networks under consideration.

Theorem 2.1. Let $(G, f)$ be a three-gene regulatory network, which has the following properties:

1. G consists of one two-gene feedback circuit, which is negative.

2. The one gene that is not in the feedback circuit is affected by the two other genes, and the influence of these two genes is governed by one of the logical gates AND, OR, or XOR.

Then the state transition graph for $(G, f)$ is connected and contains a unique attracting four-state cycle.

Proof. Let $(G, f)$ be a three-gene regulatory network, as described in the theorem statement. Since the two genes in the feedback circuit are only influenced by one another, we can consider the subnetwork created by the two-gene negative circuit independently of the third gene.

When we have a negative two-gene feedback circuit between genes $\alpha_{1}$ and $\alpha_{2}$, we observe a four-state cycle. Assuming gene $\alpha_{1}$ is an activator and $\alpha_{2}$ is an inhibitor, this subnetwork can be represented in $(G, f)$ as

$$
\begin{aligned}
& f_{1}\left(x_{1}, x_{2}\right)=1+x_{2}, \\
& f_{2}\left(x_{1}, x_{2}\right)=x_{1} .
\end{aligned}
$$

Assuming that the initial state of this subnetwork is 11 , we find the four-state cycle

$$
\begin{array}{lllll}
11 & 01 & 00 & 10 & 11 .
\end{array}
$$

Hence, the first two digits will be cycling between the four possible states for the state transition graph of the negative two-gene circuit.

Now consider the influence of the two-gene subnetwork on the gene $\alpha_{3}$. The state of the gene governed by the logical gate can then be determined by $f$ for each of the fixed states of the two-gene circuit. We first present the argument for the AND logical gate on gene $\alpha_{3}$ where both $\alpha_{1}$ and $\alpha_{2}$ are inhibitors. The state transition function takes the form

$$
\begin{aligned}
& f_{1}\left(x_{1}, x_{2}, x_{3}\right)=1+x_{2}, \\
& f_{2}\left(x_{1}, x_{2}, x_{3}\right)=x_{1} \\
& f_{3}\left(x_{1}, x_{2}, x_{3}\right)=\left(1+x_{1}\right)\left(1+x_{2}\right) .
\end{aligned}
$$

We now compute the values of the coordinate function $f_{3}$ :

$$
\begin{aligned}
& f_{3}\left(1,1, x_{3}\right)=(1+1)(1+1)=0, \\
& f_{3}\left(0,1, x_{3}\right)=(1+0)(1+1)=0, \\
& f_{3}\left(0,0, x_{3}\right)=(1+0)(1+0)=1, \\
& f_{3}\left(1,0, x_{3}\right)=(1+1)(1+0)=0 .
\end{aligned}
$$

This calculation show that the four-state cycle in the state transition graph for the two-gene subnetwork consisting of the feedback circuit between genes $\alpha_{1}$ and $\alpha_{2}$ given by

$$
\begin{array}{lllll}
11 & 01 & 00 & 10 & 11
\end{array}
$$


induces the four-state cycle

$$
\begin{array}{lllll}
110 & 010 & 000 & 101 & 110
\end{array}
$$

in the state transition graph of $(G, f)$ for the network described. Moreover, the function $f$ takes the other four states to states in this cycle.

Similar proofs work for the OR and XOR logical gates governing the behavior of the gene $\alpha_{3}$. The only differences are the specific definitions of the coordinate function $f_{3}$. In the OR case with both of $\alpha_{1}$ and $\alpha_{2}$ acting as inhibitors on $\alpha_{3}$, we have

$$
f_{3}\left(x_{1}, x_{2}, x_{3}\right)=\left(1+x_{1}\right)+\left(1+x_{2}\right)+\left(1+x_{1}\right)\left(1+x_{2}\right) .
$$

Thus

$$
\begin{aligned}
& f_{3}\left(1,1, x_{3}\right)=(1+1)+(1+1)+(1+1)(1+1)=0, \\
& f_{3}\left(0,1, x_{3}\right)=(1+0)+(1+1)+(0+1)(1+1)=1, \\
& f_{3}\left(0,0, x_{3}\right)=(1+0)+(1+0)+(1+0)(1+0)=1, \\
& f_{3}\left(1,0, x_{3}\right)=(1+1)+(1+0)+(1+1)(1+0)=1,
\end{aligned}
$$

which gives the four-state cycle

$$
111010 \quad 001 \quad 101 \quad 111 .
$$

As before, the function $f$ takes the four states that are not in the cycle to states in the cycle.

In the XOR case with both of $\alpha_{1}$ and $\alpha_{2}$ acting as inhibitors on $\alpha_{3}$, we have

$$
f_{3}\left(x_{1}, x_{2}, x_{3}\right)=\left(1+x_{1}\right)+\left(1+x_{2}\right) .
$$

Thus

$$
\begin{aligned}
& f_{3}\left(1,1, x_{3}\right)=(1+1)+(1+1)=0, \\
& f_{3}\left(0,1, x_{3}\right)=(1+0)+(1+1)=1, \\
& f_{3}\left(0,0, x_{3}\right)=(1+0)+(1+0)=0, \\
& f_{3}\left(1,0, x_{3}\right)=(1+1)+(1+0)=1,
\end{aligned}
$$

which gives the four-state cycle

$$
\begin{array}{lllll}
111 & 010 & 001 & 100 & 111 .
\end{array}
$$

The function $f$ takes the other four states to states in this cycle.

If $\alpha_{1}$ and $\alpha_{2}$ are the different combinations of activating and inhibiting influences on $\alpha_{3}$, then the function $f_{3}$ would still be a function of just $x_{1}$ and $x_{2}$, and hence we would find fourstate cycles as above for each of the three logical gates. Therefore, the state transition graph for the three-gene regulatory network $(G, f)$ is connected and contains a unique attracting four-state cycle.

Like a network with a negative circuit and the specified structure, results for a positive feedback circuit can be proved. We formally prove the results for positive circuits.

Theorem 2.2. Let $(G, f)$ be a three-gene regulatory network, which has the following properties:

1. G consists of one two-gene feedback circuit, which is positive.

2. There is exactly one gene that is not in the feedback circuit which is affected by two genes, and the influence of these genes is governed by one of the logical gates AND, OR, or XOR. 
Then the state transition graph for $(G, f)$ contains two fixed points and a unique two-state cycle.

Proof. Let $(G, f)$ be a three-gene regulatory network, as described in the theorem. Since the two genes in the feedback circuit are only influenced by one another, we can consider the subnetwork created by the two-gene positive circuit independently of the third gene.

When we have a positive two-gene feedback circuit between genes $\alpha_{1}$ and $\alpha_{2}$, we observe two fixed points and a two-state cycle. Assuming genes $\alpha_{1}$ and $\alpha_{2}$ activate one another, this subnetwork can be represented in $(G, f)$ as

$$
\begin{aligned}
& f_{1}\left(x_{1}, x_{2}\right)=x_{2}, \\
& f_{2}\left(x_{1}, x_{2}\right)=x_{1} .
\end{aligned}
$$

Hence the state transition graph for the two-gene subnetwork has the fixed points 00 and 11 and the two-state cycle

$$
01 \quad 10 \quad 01 .
$$

We now consider the influence of the two-gene subnetwork on gene $\alpha_{3}$. The state of the gene governed by the logical gate can then be determined by $f$ for each of the fixed states of the two-gene circuit. We first present the argument for the AND logical gate on gene $\alpha_{3}$ where $\alpha_{1}$ is an inhibitor and $\alpha_{2}$ is an activator. The state transition function takes the form $(G, f)$ as follows:

$$
\begin{aligned}
& f_{1}\left(x_{1}, x_{2}, x_{3}\right)=x_{2}, \\
& f_{2}\left(x_{1}, x_{2}, x_{3}\right)=x_{1}, \\
& f_{3}\left(x_{1}, x_{2}, x_{3}\right)=\left(1+x_{1}\right) x_{2} .
\end{aligned}
$$

We now compute the values of the coordinate function $f_{3}$ from the fixed points 10 and 01 of the state transition graph for the two-gene subnetwork:

$$
\begin{aligned}
& f_{3}\left(1,1, x_{3}\right)=(1+1) 1=0, \\
& f_{3}\left(0,0, x_{3}\right)=(1+0) 0=0 .
\end{aligned}
$$

Hence the fixed points 10 and 01 of the state transition graph of the two-gene subnetwork induce the fixed points

$$
000 \text { and 111, }
$$

respectively, in the state transition graph for $(G, f)$.

Computing the values of the function $f_{3}$ from the states in the two-state cycle of the state transition graph for the two-gene subnetwork, we obtain

$$
\begin{aligned}
& f_{3}\left(1,0, x_{3}\right)=(1+1) 0=0, \\
& f_{3}\left(0,1, x_{3}\right)=(1+0) 1=1 .
\end{aligned}
$$

Thus, two-state cycle of cycle of the state transition graph for the two-gene subnetwork induces the two-state cycle

$$
101 \quad 010 \quad 101
$$

in the state transition graph for $(G, f)$. Moreover, the function $f$ takes the other four states to either the fixed points or the state cycle in the state transition graph of $f$.

Similar proofs work for the OR and XOR logical gates governing the behavior of the gene $\alpha_{3}$. The only differences are the specific definitions of the coordinate function $f_{3}$. For the OR case with $\alpha_{1}$ acting as an inhibitor on $\alpha_{3}$ and $\alpha_{2}$ acting as an activator on $\alpha_{3}$, we have

$$
f_{3}\left(x_{1}, x_{2}, x_{3}\right)=\left(1+x_{1}\right)+x_{2}+\left(1+x_{1}\right) x_{2} .
$$


Thus,

$$
\begin{aligned}
& f_{3}\left(0,0, x_{3}\right)=(1+0)+0+(1+0) 0=1, \\
& f_{3}\left(1,1, x_{3}\right)=(1+1)+1+(1+1) 1=1, \\
& f_{3}\left(1,0, x_{3}\right)=(1+1)+0+(1+1) 0=0, \\
& f_{3}\left(0,1, x_{3}\right)=(1+0)+1+(1+0) 1=1,
\end{aligned}
$$

which gives the fixed points

$$
001 \text { and } 111
$$

and the two-state cycle

$$
101 \quad 010 \quad 101
$$

in the state transition graph for $(G, f)$. Once again, the function $f$ takes the remaining four states to one of the fixed points or states in the cycle.

For the XOR case with $\alpha_{1}$ acting as an inhibitor on $\alpha_{3}$ and $\alpha_{2}$ acting as an activator on $\alpha_{3}$, we have

$$
f_{3}\left(x_{1}, x_{2}, x_{3}\right)=\left(1+x_{1}\right)+x_{2} .
$$

Thus

$$
\begin{aligned}
& f_{3}\left(0,0, x_{3}\right)=(1+0)+0=1, \\
& f_{3}\left(1,1, x_{3}\right)=(1+1)+1=1, \\
& f_{3}\left(1,0, x_{3}\right)=(1+1)+0=0, \\
& f_{3}\left(0,1, x_{3}\right)=(1+0)+1=0,
\end{aligned}
$$

which gives the fixed points

$$
001 \text { and } 111
$$

and the two-state cycle

$$
100 \quad 010 \quad 100
$$

in the state transition graph for $(G, f)$. Once again, the function $f$ takes the remaining four states to one of the fixed points or states in the cycle.

If $\alpha_{1}$ and $\alpha_{2}$ inhibit each other, the state transition graph for the two-gene subnetwork consisting of the two-gene feedback circuit containing $\alpha_{1}$ and $\alpha_{2}$ has fixed points 10 and 01 and the two-state cycle

$$
11 \quad 00 \quad 11 .
$$

For the cases considered above with $\alpha_{1}$ inhibiting $\alpha_{3}$ and $\alpha_{2}$ activating $\alpha_{3}$, we similarly obtain state transition graphs for $(G, f)$ with two fixed points and a unique two-state cycle.

Furthermore, observe that the function $f_{3}$ would remain a function of just $x_{1}$ and $x_{2}$ even if the manners in which $\alpha_{1}$ and $\alpha_{2}$ influence $\alpha_{3}$ were different. Therefore, in all possible cases, the state transition graph for the three-gene regulatory network $(G, f)$ has two fixed points and a unique two-state cycle.

\section{Continuous Model}

The concentrations of the macromolecules involved with gene expression can be represented using differential equations. A system of equations for the mRNA, $R_{i}$, and the protein $P_{i}$ produced by each gene $\alpha_{i}$ can be written for each interaction graph. Assuming we use the same structure of the interaction graph of the gene network, where $\alpha_{1}$ and $\alpha_{2}$ are in 
a feedback circuit and $\alpha_{3}$ is influenced by both $\alpha_{1}$ and $\alpha_{2}$, governed by a logical gate, the system of equations are of the following form:

$$
\begin{array}{ll}
\text { when one gene is influencing a gene } & \dot{R}_{1}=r m_{1} F_{1}\left(P_{2}\right) \quad r \gamma_{1} R_{1}, \\
& \dot{R}_{2}=r m_{2} F_{2}\left(P_{1}\right) \quad r \gamma_{2} R_{2}, \\
\text { when two genes are influencing a gene } & \dot{R}_{3}=r m_{3} F_{3}\left(P_{1}, P_{2}\right) \quad r \gamma_{3} R_{3}, \\
\text { protein expression for all genes } & \dot{P}_{1}=\omega_{1} R_{1} \quad \delta_{1} P_{1}, \\
& \dot{P}_{2}=\omega_{2} R_{2} \quad \delta_{2} P_{2}, \\
& \dot{P}_{3}=\omega_{3} R_{3} \quad \delta_{3} P_{3} .
\end{array}
$$

where the translation and degradation rates are denoted by $\omega_{i}$ and $\gamma_{i}$, respectively. Additionally, the maximal transcription rate of proteins, $m_{i}$, and degradation rate of $\delta_{i}$ are represented in the model. A time scale ratio of mRNA to protein production is defined by $\frac{R_{i}}{P_{i}}=r$, also. As with the Boolean framework, logical gates can be accounted for in the continuous model; these are introduced using Hill functions. The functions $F_{n}$ are Hill functions, switch-like functions that correspond to a gene being either ON or OFF. The following functions can be defined for gene $i$ that is influenced by gene(s) $j$ :

$$
\begin{aligned}
& F_{i}^{+}\left(P_{j}, k_{j}, n_{j}\right)=\frac{P_{j}^{n_{j}}}{P_{j}^{n_{j}}+k_{j}^{n_{j}}} \\
& F_{i}^{-}\left(P_{j}, k_{j}, n_{j}\right)=1 \quad F_{i}^{+}\left(P_{j}, k_{j}, n_{j}\right)=\frac{k_{j}^{n_{j}}}{P_{j}^{n_{j}}+k_{j}^{n_{j}}} .
\end{aligned}
$$

In the Hill functions, $k$ is the expression threshold of the gene, and $n$ is the Hill coefficient, which biologically corresponds to the number of binding sites present in the gene. We use $F^{+}$when the gene is an activator and $F^{-}$when the gene is an inhibitor. Using the Hill functions, we can construct our system of equations according to the interaction graph of the desired network. Logical gates can be represented by the addition and multiplication of these Hill functions. As with the Boolean model, when a gene is influenced by two genes, the logical gates must be considered.

In order to represent the logical gates governing a gene, the composition of Hill functions is used. Consider a network in which gene $\alpha_{3}$ is influenced by genes $\alpha_{1}$ and $\alpha_{1}$. Additionally, let $\alpha_{1}$ be an activator and $\alpha_{2}$ be an inhibitor, then the following functions for the corresponding logical gates can be constructed:

$$
\begin{aligned}
& \text { AND: } \quad \dot{R}_{3}=r m_{3}\left[\left(\frac{P_{1}^{n}}{P_{1}^{n}+k_{1}^{n}}\right)\left(\frac{k_{2}^{n}}{P_{2}^{n}+k_{2}^{n}}\right)\right] \quad r \gamma_{3} R_{3}, \\
& \text { XOR: } \quad \dot{R}_{3}=r m_{3}\left[\left(\frac{P_{1}^{n}}{P_{1}^{n}+k_{1}^{n}}\right)+\left(\frac{k_{2}^{n}}{P_{2}^{n}+k_{2}^{n}}\right)\right] \quad r \gamma_{3} R_{3} \text {, }
\end{aligned}
$$

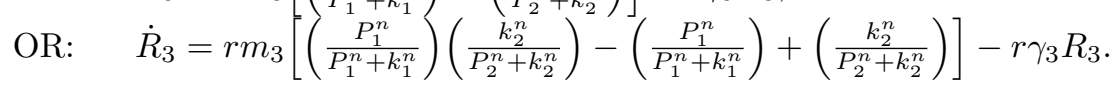

Because the degradation, transcription, and translation rates will be approximately the same at all times, these parameter values remain constant. However, the time scale ratio $r$ of the concentration of mRNA to protein would vary for a network, in the proposed model it is varied between $r=1$ and $r=50$. Similarly, the Hill coefficients would be allowed to vary between the values $n=2,5,10$ to test the continuous dynamics. Examples of what the dynamics of the continuous model looks like for a gene regulatory network for the different parameter values can be seen in Figures 3, 4, and 5. Table 1 summarizes the dynamics of the continuous model in terms of the structure of the interaction graphs.

The continuous dynamics for these small gene regulatory networks in Table 1 show that when there is a positive circuit present in the interaction graph, stabilizing behavior always occur. Although some oscillations may be observed in networks with a positive feedback circuit, as time progresses there is always stabilizing behavior. When a negative feedback circuit is present in the interaction graph, there is always eventually oscillatory behavior. As the parameters are increased, the networks always show oscillatory behavior. These results from Table 1 can be compared with results from the Boolean model to determine if there is an underlying relationship among these models. 


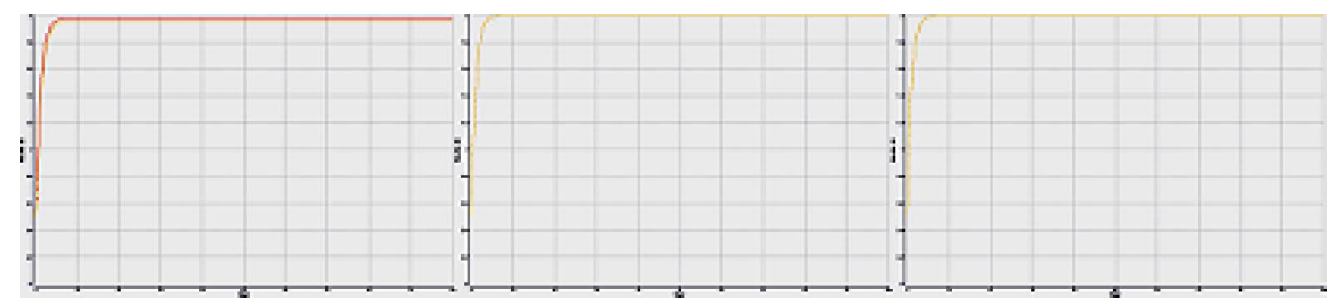

Figure 3: Time series for the continuous model of a three-gene regulatory network with a positive feedback circuit, where $r=1$ and, from the left to right, $n=2,5,10$.

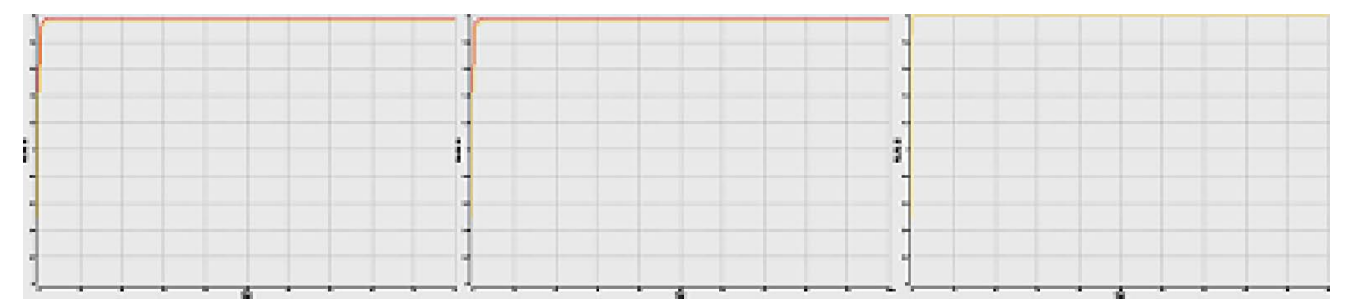

Figure 4: Time series for the continuous model of a three-gene regulatory network with a positive feedback circuit, where $r=50$ and, from the left to right, $n=2,5,10$.

\section{Analysis}

To better understand when the continuous model exhibits either oscillatory or stable behavior, we determine where Hopf bifurcations occur in the parameter space. Following Gehrmann and Drossel [4], we use the technique of generalized models due to Gross and Feudal [6] to normalize our model and then the method of resultants also due to Gross and Feudal [5] to determine where the bifurcations occur. The technique of generalized models allows us to address different logical functions for $F_{3}\left(P_{a}, P_{b}\right)$ and parameter values at once and allows us to study different dynamical regions without the need to specify the form of the reaction or the specific equilibrium concentrations.

We consider the general gene regulatory network shown in Figure 6 in which we do not $a$ priori specify whether influences are activating or inhibitory. The structure of the analysis will be the same regardeless of the types of gene interactions occuring. To begin the process of determining where the model exhibits oscillatory behavior, we perform linear stability analysis of the fixed points and determine where a Hopf bifurcation occurs. The fixed points are obtained by setting the six derivatives in the model equal to zero and then by solving for the fixed points of the form

$$
\left(R_{1}^{*}, R_{2}^{*}, R_{3}^{*}, P_{1}^{*}, P_{2}^{*}, P_{3}^{*}\right)
$$

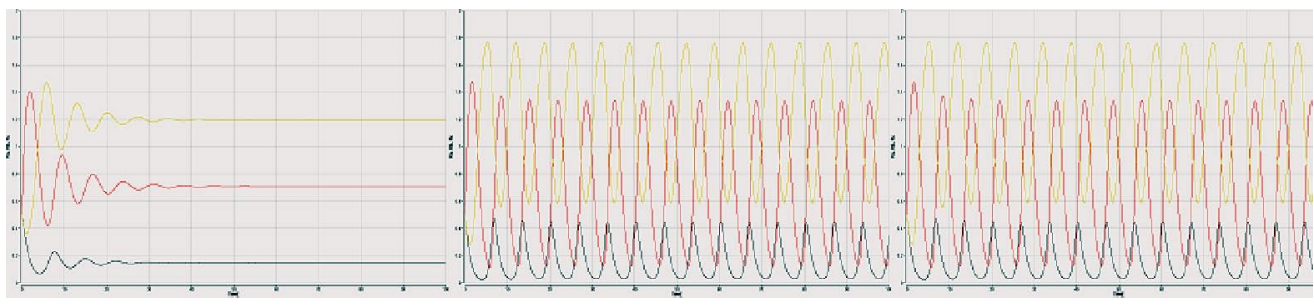

Figure 5: Time series for the continuous model of a three-gene regulatory network with a negative feedback circuit, where $r=1$ and, from the left to right, $n=2,5,10$. 
Table 1: Continuous dynamics for small gene regulatory networks based on network characteristics

\begin{tabular}{|c|l|}
\hline Sign of Feedback Circuit & Dynamics \\
\hline Positive Circuit & $\begin{array}{l}\text { Stabilizing occurs; occasional oscillations be- } \\
\text { fore stabilizing }\end{array}$ \\
\hline Negative Circuit & Eventually see oscillatory behavior \\
\hline
\end{tabular}

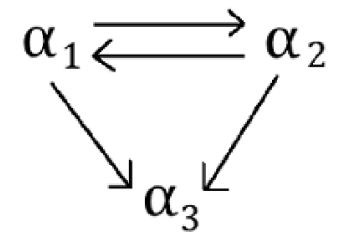

Figure 6: General interaction graph for a three-gene regulatory network.

We obtain the equations

$$
\begin{gathered}
R_{1}^{*}=\frac{\delta_{1}}{\omega_{1}} P_{1}^{*}, \quad R_{2}^{*}=\frac{\delta_{2}}{\omega_{2}} P_{2}^{*}, \quad R_{3}^{*}=\frac{\delta_{3}}{\omega_{3}} P_{3}^{*}, \\
P_{1}^{*}=\frac{\gamma_{1} \delta_{1}}{\omega_{1} m_{1}} F_{1}\left(P_{2}\right), \quad P_{2}^{*}=\frac{\gamma_{2} \delta_{2}}{\omega_{2} m_{2}} F_{2}\left(P_{1}\right), \quad P_{3}^{*}=\frac{\gamma_{3} \delta_{3}}{\omega_{3} m_{3}} F_{3}\left(P_{1}, P_{2}\right) .
\end{gathered}
$$

The Jacobian of the system at the fixed point can be written as

$$
\begin{aligned}
& J=\left(\begin{array}{cccccc}
-r \gamma_{1} & 0 & 0 & 0 & r m_{1} \frac{\partial F_{1}}{\partial P_{2}} & 0 \\
0 & -r \gamma_{2} & 0 & r m_{2} \frac{\partial F_{2}}{\partial P_{1}} & 0 & 0 \\
0 & 0 & -r \gamma_{c} & r m_{3} \frac{\partial F_{3}}{\partial P_{1}} & r m_{3} \frac{\partial F_{3}}{\partial P_{2}} & 0 \\
\omega_{1} & 0 & 0 & -\delta_{1} & 0 & 0 \\
0 & \omega_{2} & 0 & 0 & -\delta_{2} & 0 \\
0 & 0 & \omega_{3} & 0 & 0 & -\delta_{3}
\end{array}\right) \\
& =\left(\begin{array}{cc}
\text { mRNA degradation } & \text { transcription } \\
\text { translation } & \text { protein degradation }
\end{array}\right) \text {. }
\end{aligned}
$$

To begin the process of using generalized models, we assume that there is at least one positive equilibrium

$$
\left(R_{1}^{*}, R_{2}^{*}, R_{3}^{*}, P_{1}^{*}, P_{2}^{*}, P_{3}^{*}\right) .
$$

We now define the normalized variables

$$
r_{1}=\frac{R_{1}}{R_{1}^{*}}, \quad r_{2}=\frac{R_{2}}{R_{2}^{*}}, \quad r_{3}=\frac{R_{3}}{R_{3}^{*}}, \quad p_{1}=\frac{P_{1}}{P_{1}^{*}}, \quad p_{2}=\frac{P_{2}}{P_{2}^{*}}, \quad p_{3}=\frac{P_{3}}{P_{3}^{*}},
$$

which give rise to the normalized functions

$$
\tilde{f}_{j}\left(p_{i}\right)=\frac{F_{j}\left(P_{i}^{*} p_{i}\right)}{F_{j}^{*}}, \quad \text { where } \quad i=1,2,3
$$

and

$$
F_{1}^{*}=F_{1}\left(P_{2}\right), \quad F_{2}^{*}=F_{2}\left(P_{1}\right), \quad F_{3}^{*}=F_{3}\left(P_{1}, P_{2}\right) .
$$


The normalized system is

$$
\begin{array}{rllll}
r_{1}^{\prime} & =\frac{m_{1} F_{1}^{*} r}{R_{1}^{*}} \tilde{f}_{1}\left(p_{1}\right) & \gamma_{1} r_{1} r, & p_{1}^{\prime}=\frac{R_{1}^{*}}{P_{1}^{*}} \omega_{1} r_{1} & \delta_{1} p_{1}, \\
r_{2}^{\prime} & =\frac{m_{2} F_{2}^{*} r}{R_{2}^{*}} \tilde{f}_{2}\left(p_{2}\right) & \gamma_{2} r_{2} r, & p_{2}^{\prime}=\frac{R_{2}^{*}}{P_{2}^{*}} \omega_{2} r_{2} & \delta_{2} p_{2}, \\
r_{3}^{\prime} & =\frac{m_{3} F_{3}^{*} r}{R_{3}^{*}} \tilde{f}_{3}\left(p_{1}, p_{2}\right) & \gamma_{3} r_{3} r, & p_{3}^{\prime}=\frac{R_{3}^{*}}{P_{3}^{*}} \omega_{3} r_{3} & \delta_{3} p_{3} .
\end{array}
$$

The normalized fixed point becomes

$$
\left(r_{1}^{*}, r_{2}^{*}, r_{3}^{*}, p_{1}^{*}, p_{2}^{*}, p_{3}^{*}\right)=(1,1,1,1,1,1) .
$$

To further simplify the model, we introduce the new parameters

$$
\begin{array}{ll}
\alpha_{r}=\frac{m_{1} F_{1}^{*} r}{R_{1}^{*}}=\gamma_{1} r, & \alpha_{p}=\frac{R_{1}^{*} \omega_{1}}{P_{1}^{*}}=\delta_{1}, \\
\beta_{r}=\frac{m_{2} F_{2}^{*} r}{R_{2}^{*}}=\gamma_{2} r, & \beta_{p}=\frac{R_{2}^{*} \omega_{2}}{P_{2}^{*}}=\delta_{2}, \\
\mu_{r}=\frac{m_{3} F_{3}^{*} r}{R_{3}^{*}}=\gamma_{3} r, & \mu_{p}=\frac{R_{3}^{*} \omega_{3}}{P_{3}^{*}}=\delta_{3} .
\end{array}
$$

The model can now be expressed as

$$
\begin{aligned}
& r_{1}^{\prime}=\alpha_{r}\left(\tilde{f}_{1}\left(p_{2}\right) \quad r_{1}\right), \quad p_{1}^{\prime}=\alpha_{p}\left(\begin{array}{ll}
r_{1} & p_{1}
\end{array}\right), \\
& r_{2}^{\prime}=\beta_{r}\left(\tilde{f}_{2}\left(p_{1}\right) \quad r_{2}\right), \quad p_{2}^{\prime}=\beta_{p}\left(\begin{array}{ll}
r_{2} & p_{2}
\end{array}\right), \\
& r_{3}^{\prime}=\mu_{r}\left(\tilde{f}_{3}\left(p_{1}, p_{2}\right) \quad r_{3}\right), \quad p_{3}^{\prime}=\mu_{p}\left(\begin{array}{ll}
r_{3} & p_{3}
\end{array}\right) .
\end{aligned}
$$

In this generalized model, $\alpha_{i}, \beta_{i}$, and $\gamma_{i}$ are measures of the (inverse) time scales of the mRNA and protein concentrations. We assume that the time scale ratio is

$$
r=\frac{\alpha_{r}}{\alpha_{p}}=\frac{\beta_{r}}{\beta_{p}}=\frac{\mu_{r}}{\mu_{p}} .
$$

The Jacobian of the normalized model becomes

$$
\bar{J}=\left(\begin{array}{cccccc}
\alpha_{r} & 0 & 0 & 0 & 0 & 0 \\
0 & \beta_{r} & 0 & 0 & 0 & 0 \\
0 & 0 & \mu_{r} & 0 & 0 & 0 \\
0 & 0 & 0 & \alpha_{p} & 0 & 0 \\
0 & 0 & 0 & 0 & \beta_{p} & 0 \\
0 & 0 & 0 & 0 & 0 & \mu_{p}
\end{array}\right)\left(\begin{array}{cccccc}
1 & 0 & 0 & \frac{\partial \tilde{f}_{1}}{\partial p_{1}} & \frac{\partial \tilde{f}_{1}}{\partial p_{2}} & \frac{\partial \tilde{f}_{1}}{\partial p_{3}} \\
0 & 1 & 0 & \frac{\partial \tilde{f}_{2}}{\partial p_{1}} & \frac{\partial \tilde{f}_{2}}{\partial p_{2}} & \frac{\partial f_{2}}{\partial p_{3}} \\
0 & 0 & 1 & \frac{\partial \tilde{f}_{1}}{\partial p_{1}} & \frac{\partial \tilde{f}_{1}}{\partial p_{2}} & \frac{\partial \tilde{f}_{1}}{\partial p_{3}} \\
1 & 0 & 0 & 1 & 0 & 0 \\
0 & 1 & 0 & 0 & 1 & 0 \\
0 & 0 & 1 & 0 & 0 & 1
\end{array}\right) .
$$

By substituting $r$ for the timescale ratio and noting that the $A, B, C$, and $D$ represent the only possible nonzero partial derivatives, the Jacobian becomes

$$
\bar{J}=\left(\begin{array}{llllll}
r & 0 & 0 & 0 & 0 & 0 \\
0 & r & 0 & 0 & 0 & 0 \\
0 & 0 & r & 0 & 0 & 0 \\
0 & 0 & 0 & 1 & 0 & 0 \\
0 & 0 & 0 & 0 & 1 & 0 \\
0 & 0 & 0 & 0 & 0 & 1
\end{array}\right)\left(\begin{array}{cccccc}
1 & 0 & 0 & 0 & A & 0 \\
0 & 1 & 0 & B & 0 & 0 \\
0 & 0 & 1 & C & D & 0 \\
1 & 0 & 0 & 1 & 0 & 0 \\
0 & 1 & 0 & 0 & 1 & 0 \\
0 & 0 & 1 & 0 & 0 & 1
\end{array}\right) .
$$

We are now in the position to determine the eigenvalues of $\bar{J}$. We look for two particular cases. A Hopf bifurcation occurs when a pair of symmetric purely imaginary eigenvalues 
occur. This bifurcation is an indication of stable oscillatory behavior on one side of the bifurcation and stable equilibria on the the other side. A real Hopf situation occurs when there is a pair of symmetric real eigenvalues. This does not indicate an actual change in the dynamics of the system. We complete the analysis using the method of resultants [5] to determine the bifurcation surfaces.

We first find the characteristic polynomial of $\bar{J}$, which is

$$
c_{6} \lambda^{6}+c_{5} \lambda_{5}+\quad+c_{1} \lambda+c_{0},
$$

where

$$
\begin{array}{lll}
c_{0}=r^{3} \quad r^{3} A B, & c_{1}=r A B+3 r^{2}+3 r^{3} \quad r^{3} A B, \\
c_{2}=3 r+9 r^{2}+3 r^{3} & r^{2} A B, & c_{3}=r^{3}+9 r^{2}+9 r+1, \\
c_{4}=3 r^{2}+9 r+3, & c_{5}=3 r+3, \\
c_{6}=1 . &
\end{array}
$$

We then find the resultant determinant

$$
R=\left|\begin{array}{ccccc}
c_{1} & c_{0} & 0 & 0 & 0 \\
c_{3} & c_{2} & c_{1} & c_{0} & 0 \\
c_{5} & c_{4} & c_{3} & c_{2} & c_{1} \\
0 & c_{6} & c_{5} & c_{4} & c_{3} \\
0 & 0 & 0 & c_{6} & c_{5}
\end{array}\right| .
$$

In factored form, the resultant is

$$
R=4 r^{3}(1+r)^{3}\left(A B \quad 4 \quad 8 r \quad 4 r^{2}\right)\left(r^{2} A B \quad 4 \quad 8 r \quad 4 r^{2}\right)\left(r A B+1+2 r+r^{2}\right) .
$$

The bifurcation manifold obtained from this resultant in the three parameter $(r, B, A)$ space is implicitly given by

$$
4 r^{3}(1+r)^{3}\left(A B \quad 4 \quad 8 r \quad 4 r^{2}\right)\left(r^{2} A B \quad 4 \quad 8 r \quad 4 r^{2}\right)\left(r A B+1+2 r+r^{2}\right)=0 .
$$

Now the factor $\left(r A B+1+2 r+r^{2}\right)$ corresponds to the Hopf bifurcation because $\bar{J}$ possess a pair of symmetric, purely imaginary eigenvalues. The corresponding surface is plotted in Figure 7. Oscillatory behavior occurs in the components of the parameter space bounded by the two branches of this surface not containing $(0,0,0)$. The component containing $(0,0,0)$ corresponds to a stable fixed point. From the figure, we see that Hopf bifurcations and hence oscillatory behavior occur when $A$ and $B$ have opposite signs. This make sense and agrees with the results of Gehrmann and Drossel [4] because this indicates the existence of an activitor-inhibitor relationship between genes $\alpha_{1}$ and $\alpha_{2}$. Additionally, the figure indicates that region for which oscillatory behavior occurs shrinks as the time scale ratio $r$ increases. The factors $\left(\begin{array}{lllllll}r^{2} A B & 4 & 8 r & 4 r^{2}\end{array}\right)$ and $\left(\begin{array}{lllll}A B & 4 & 8 r & 4 r^{2}\end{array}\right)$ correspond to real Hopf situations because $\bar{J}$ contains a pair of real symmetric eigenvalues. These branches meet when $r=1$ and are plotted in Figure 8. The Hopf bifurcation surface and the real Hopf situation surfaces do not intersect.

We now summarizes the results of this section with the following theorem.

Theorem 4.1. Consider a gene regulatory network whose wiring diagram is Figure 6 and whose dynamics are described by the normalized system (3). Let $A=\frac{\partial \tilde{f}_{1}}{\partial p_{2}}$ and $B=\frac{\partial \tilde{f}_{2}}{\partial p_{1}}$.

1. In the $(r, A, B)$-parameter space, the system exhibits a Hopf bifurcation along the surface given by

$$
r A B+1+2 r+r^{2}=0 .
$$

The region between the two branches of this surface containing $(0,0,0)$ corresponds to the existence of a stable fixed point for the system. The other two regions correspond to oscillatory behavior. 


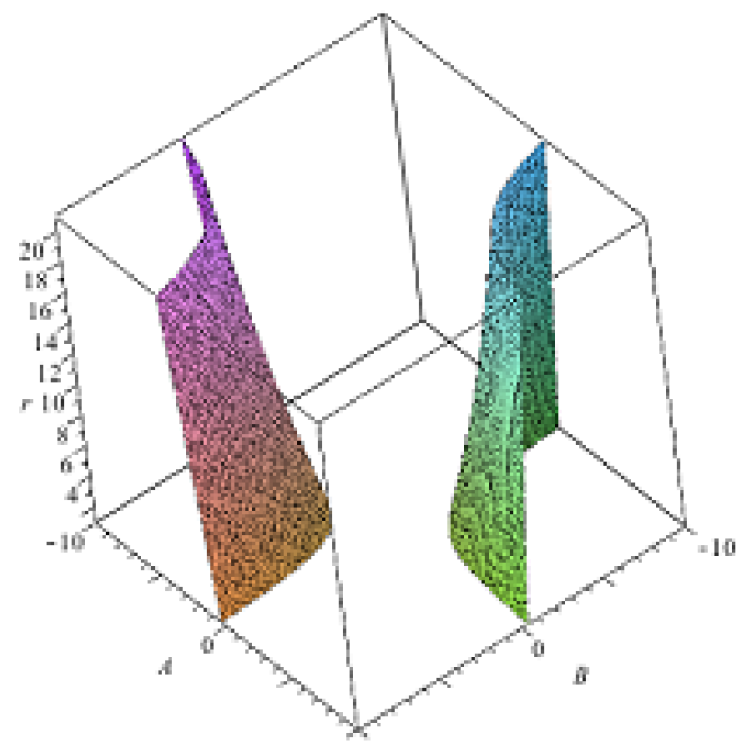

Figure 7: The Hopf bifurcation surface

2. In the $(r, A, B)$-parameter space, the system exhibits a real Hopf situation along the intersecting surfaces given by

$$
r^{2} A B-4-8 r-4 r^{2}=0 \quad \text { and } \quad A B-4-8 r-4 r^{2}=0 .
$$

\section{Discussion}

The original goal of our investigation was to determine if there was a connection between the Boolean and continuous models of small gene regulatory networks. When first comparing the qualitative behavior of these small networks, there appeared to be no clear relationship between the Boolean and continuous models. However, we were able to conclude that a state cycle in the Boolean model does not imply that there will be oscillations in the continuous model. This agreed with previous work comparing Boolean and continuous models of twogene regulatory networks [4].

Since a clear connection between the two models was not evident, stability analysis of the continuous model was performed. We determined when a Hopf bifurcation occurs for our gene regulatory networks, which represents the continuous model switching dynamical behavior. More precisely, Hopf bifurcations showed where the Boolean and continuous models switched from disagreement to agreement. Below is a table of all the results for all parts of the study conducted in order to see the entirety of the work together. Table 2 allows an easy comparison of the dynamics of the networks with respect to the sign of feedback circuits present in the networks.

By determining the occurrence of Hopf bifurcations, we were able to find when the dynamical behavior of the two models agreed. In the future, we plan to continue comparing the qualitative dynamics of the Boolean and continuous models. In addition, we plan to continue looking at more complex small gene regulatory networks and eventually larger networks.

\section{Acknowledgments}

We thank the referees for their helpful comments. 


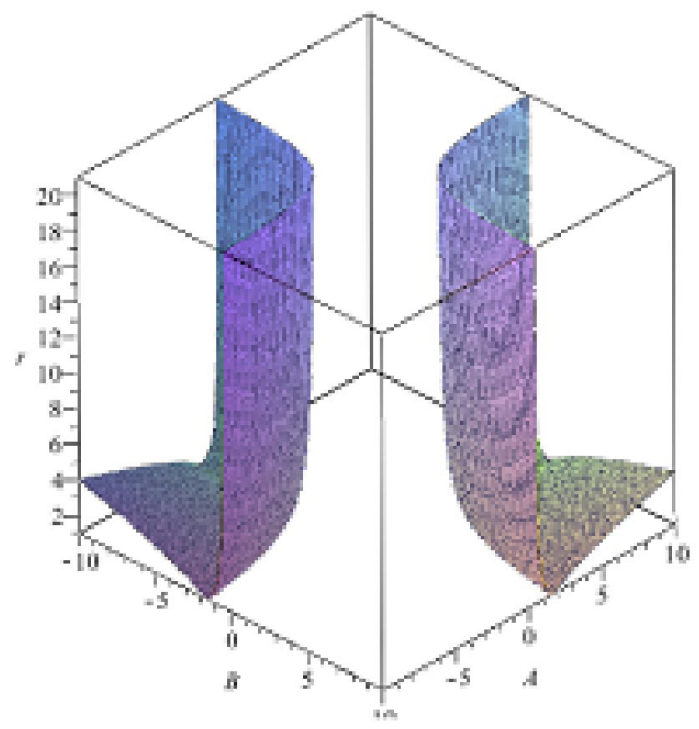

Figure 8: The real Hopf situation surface

Table 2: Dynamics of each analysis for small gene regulatory networks based on network characteristics

\begin{tabular}{|l|l|l|l|}
\hline $\begin{array}{l}\text { Feedback } \\
\text { Circuits }\end{array}$ & $\begin{array}{l}\text { Boolean } \\
\text { Dynamics }\end{array}$ & $\begin{array}{l}\text { Continuous } \\
\text { Dynamics }\end{array}$ & $\begin{array}{l}\text { Hopf } \\
\text { Dynamics }\end{array}$ \\
\hline $\begin{array}{l}\text { Positive } \\
\text { Circuit }\end{array}$ & $\begin{array}{l}\text { Two-state } \\
\text { cycles and } \\
\text { fixed points }\end{array}$ & $\begin{array}{l}\text { Stabilizing be- } \\
\text { haviors with some } \\
\text { oscillations before } \\
\text { stabilizing }\end{array}$ & $\begin{array}{l}\text { Occur when } \\
r \text { and }\end{array}$ \\
\hline $\begin{array}{l}\text { Negative } \\
\text { Circuits }\end{array}$ & $\begin{array}{l}\text { Two- aventually have } \\
\text { four-state } \\
\text { cycles }\end{array}$ & $\begin{array}{l}\text { Oscillatory behav- } \\
\text { ior }\end{array}$ & \begin{tabular}{l}
$r<6$ \\
\hline
\end{tabular}
\end{tabular}

\section{References}

[1] D. Bollman, O. Colon-Reyes, and E. Orozco. 2007. Fixed points in discrete models for regulatory genetic networks. EURASIP JBSB. 2007 (97356): 1-8.

[2] D. Del Vecchio and E. D. Sontag. 2007. Dynamics and control of synthetic biomolecular networks. Proceedings of American Control Conference, New York. 1577-1588.

[3] G. de Vries, T. Hillen, M. Lewis, H. Muller, and B. Schonfisch. 2006. A course in mathematical biology. Philadelphia, PA. 86.

[4] E. Gehrmann and B. Drossel. 2010. Boolean versus continuous dynamics on simple two-gene modules. Phys Rev E. 82 (046120): 1-9.

[5] T. Gross and U. Feudel. 2004. Analytical search for bifurcation sufraces in parameter space. Physica D 195: 292-302.

[6] T. Gross and U. Feudel. 2006. Generalized models as a universal approach to the analysis of nonlinear dynamical systems. Phys Rev E. 73 (016295): 1-14.

[7] R. Guantes and J. F. Poyatos. 2006. Dynamical Principles of Two-Component Genetic Oscillators. PLOS Comput. Biol. 2, e30. 
[8] A. Mochizuki. 2005. An analytical study of the number of steady states in gene regulatory networks. J. Theor. Biol., 236, 291-310.

[9] J. Norrell, B. Samuelsson, and J. E. Socolar. 2007. Attractors in continuous and Boolean networks. Phys. Rev. E. 76(4 Pt 2):046122. Epub 2007 Oct 30.

[10] A. Polynikis, S. J. Hogan, and M. di Bernardo. 2009. Comparing different ODE modelling approaches for gene regulatory networks. J. Theor. Biol. 251(4), 511-530.

[11] H. Siebert. 2011. Analysis of discrete bioregulatory networks using symbolic steady states. Bull Math Biol. 2010 (73): 873-898.

[12] H. Siebert and A. Bockmayr. 2007. Relating Attractors and Singular States in the Logical Analysis of Bioregulatory Networks. In LNCS: Vol. 4545. Algebraic biology, AB 2007, Castle of Hagenberg, Austria, 36-50. Berlin, Springer.

[13] D. Thieffry, E. Remy, and P. Ruet. 2008. Graphic requirements for multistability and attractive cycles in a Boolean dynamical framework. Advances in Applied Mathematics, 41(3): $335-350$

[14] R. Thomas. 1981. On the relation between the logical structure of systems and their ability to generate multiple steady states or sustained oscillations. Springer series in Synergetic. 9: 180-193.

[15] R. Thomas, D. Theiffry, and M. Kaufman. 1995. Dynamical behavior of biological regulatory networks I. biological role of feedback loops and practical use of the concept of the loop-characteristic state. Bull Math Bio. 57: 247-276.

[16] A. Veliz-Cuba and B. Stigler. 2011. Boolean models can explain bistability in the lac operon. J Comput Biol. 18 (6): 783-794.

[17] S. Widder, J. Schicho, and P. Schuster. 2007 Dynamic patterns of gene regulation I: Simple two-gene systems. J. Theor. Biol. 246(3), 395-419.

[18] D. M. Wittmann, J. Krumsiek, J. Saez-Rodriguez, D. A. Lauffenburger, S. Klamt, and F. J. Theis. 2009. Transforming Boolean models to continuous models: methodology and application to T-cell receptor signaling. BMC Syst Biol. 3 (98): 1-28. 